\title{
Emprego de Spray Dryer na indústria de alimentos: Uma breve revisão
}

\author{
Bruno Engel ${ }^{1}$ \\ Nádia de Monte Baccar ${ }^{2}$ \\ Liliane Marquardt ${ }^{3}$ \\ Ana Lúcia Becker Rohlfes ${ }^{2}$
}

\section{RESUMO}

A secagem é um processo importante e amplamente empregado para a conservação de diversos tipos de alimentos. Quando realizado com o auxílio de elevadas temperaturas e tempos de secagem, pode ocasionar danos nos constituintes dos alimentos, como a degradação de componentes bioativos. O spray dryer é uma das técnicas de secagem que se destacam na indústria alimentícia, por ser um processo rápido, contínuo, econômico e por não expor o produto a elevadas temperaturas, diminuindo desta forma a sua degradação e perdas nutricionais. As propriedades do produto formado no processo dependem de uma série de variáveis que devem ser controladas, como a propriedades do líquido de alimentação, o design do equipamento e os parâmetros do processo. Estudos demonstraram que em condições controladas, esta técnica é eficaz para diversos tipos de alimentos. Neste sentido, este presente trabalho objetivou-se na revisão bibliográfica de aspectos importantes presentes no uso do processo de secagem por spray dryer na indústria alimentícia.

Palavras-chave: Alimentos. Secagem. Spray Dryer. Indústria Alimentícia.

\section{ABSTRACT}

Drying is an important and widely used process for the conservation of various types of food. When performed with high temperatures and drying times, it can cause damage to food constituents, such as degradation of bioactive components. Spray dryer is one of the most used drying techniques in the food industry, because it's rapid, continuous, cost-effective and does not expose the product to high temperatures, reducing in this way its degradation and nutritional losses. The properties of the product formed in the process depend on a number of variables that must be controlled, such as feed liquid properties, equipment design and process parameters. Studies demonstrate that under controlled conditions, this technique is effective for various types of food. In this sense, this present work was aimed at the review of important aspects present in the use of the drying process by spray dryer in the food industry.

Keywords: Food. Dehydration. Spray Dryer. Food Industry.

\footnotetext{
${ }^{1}$ Acadêmico do curso de Química da Universidade de Santa Cruz do Sul - UNISC.

${ }^{2}$ Professoras do Departamento de Química e Física na Universidade de Santa Cruz do Sul. <albecker@unisc.br> ${ }^{3}$ Professora do Departamento de Engenharia, Arquitetura e Ciências Agrárias na Universidade de Santa Cruz do Sul.
} 


\section{INTRODUÇÃO}

A secagem é uma das técnicas mais antigas destinadas à preservação dos alimentos, atuando não apenas na remoção da água, como também sendo responsável por mudanças em aspectos físico-químicos e biológicos do produto. Dentre os benefícios destacam-se o aumento da vida útil do produto, preservação do valor nutricional, facilidade de transporte e comercialização e redução de perdas pós-colheita (CELESTINO, 2010; DADALI; DEMIRHAN; ÖZBEK, 2008).

Há diversas técnicas diferentes para a secagem desses produtos, incluindo secadores em bandeja, túnel, tambor, fluidização e Spray Dryer, responsáveis por remover uma grande quantidade de água dos produtos e que requerem muita energia durante o processo (CAGLAYAN; CALISKAN, 2017). A desidratação por meio de aquecimento, dependendo do tempo e das temperaturas de exposição, podem ocasionar alterações sensoriais e nutricionais ao produto, incluindo a degradação de componentes importantes como as vitaminas (CELESTINO, 2010; CORREIA; FARAONI; PINHEIRO SANT’ANA, 2008). Por outro lado, a desidratação é apontada como um dos procedimentos mais importantes para a diminuição da atividade de água (aw). Comparada com outros métodos preservativos para períodos longos, como a centrifugação, o enlatamento, os tratamentos químicos, a irradiação, entre outros, é de custo mais baixo e de operação mais simples (ALEXANDRE et al., 2009)

Muitos dos produtos alimentícios podem ser secos na forma de um pó, para proporcionar maior vida útil e para proteger a atividade de componentes bioativos. Entre os métodos convectivos de secagem com ar, o Spray Dryer é considerado uma técnica interessante devido aos seus tempos de secagem muito curtos e às temperaturas relativamente baixas em que o produto é exposto. Devido a essas características, a técnica é amplamente utilizada em várias áreas da indústria, incluindo a de alimentos (SCHUTYSER; PERDANA; BOOM, 2012; MASTERS, 1972).

A técnica de Spray Dryer é bem estabelecida e tem sido utilizada há mais de um século, mas continua sendo um campo ativo de inovação, impulsionada pela crescente demanda de partículas mais sofisticadas (VEHRING; FOSS; LECHUGA-BALLESTEROS, 2007). Muitos trabalhos vêm sendo realizados buscando condições ideais e específicas para a secagem de grãos, a produção de pó de frutas e produtos vegetais (SOUZA, 2014; TONON, BRADET; HUBINGER, 2013; PENG et al., 2013; SILVA et al., 2015). Também se destaca o uso do Spray Dryer para a indústria de lacticínios (PERRONE, PEREIRA, CARVALHO, 2011; TRIBOLI et. al., 2014). 
Considerando-se a necessidade do emprego de técnicas rápidas e que não deteriorem os componentes durante a obtenção de diversos produtos alimentícios, o presente trabalho apresenta uma breve revisão de aspectos importantes da técnica de Spray Dryer voltada para a indústria de alimentos.

\section{Spray drying}

A secagem por atomização, também conhecida como spray dryer, vem sendo utilizada há décadas, com descrições que datam de 1860 e oficialização da primeira patente no ano de 1872. O Spray Dryer é uma técnica de custo relativamente baixos, o que atrai o interesse da indústria, inclusive a de alimentos (DZIEZAK, 1987; SANTOS et al., 2014).

O esquema de funcionamento do equipamento é apresentado na Figura 1. A amostra utilizada na alimentação (1) pode estar em solução, suspenção, emulsão ou na forma de pasta. Com auxílio de uma bomba, a amostra é carregada até um atomizador (2) que transforma o líquido em pequenas gotículas que entram em contato com o ar aquecido (3) no interior da câmara de secagem (4). O ar aquecido remove a água da gotícula, resultando em um pó fino, do produto, que passa por um ciclone (6) e é acondicionado em um coletor (7) (AZEREDO, 2004; KESHANI et al., 2015).

Figura 1 - Esquema de funcionamento do Spray Dryer

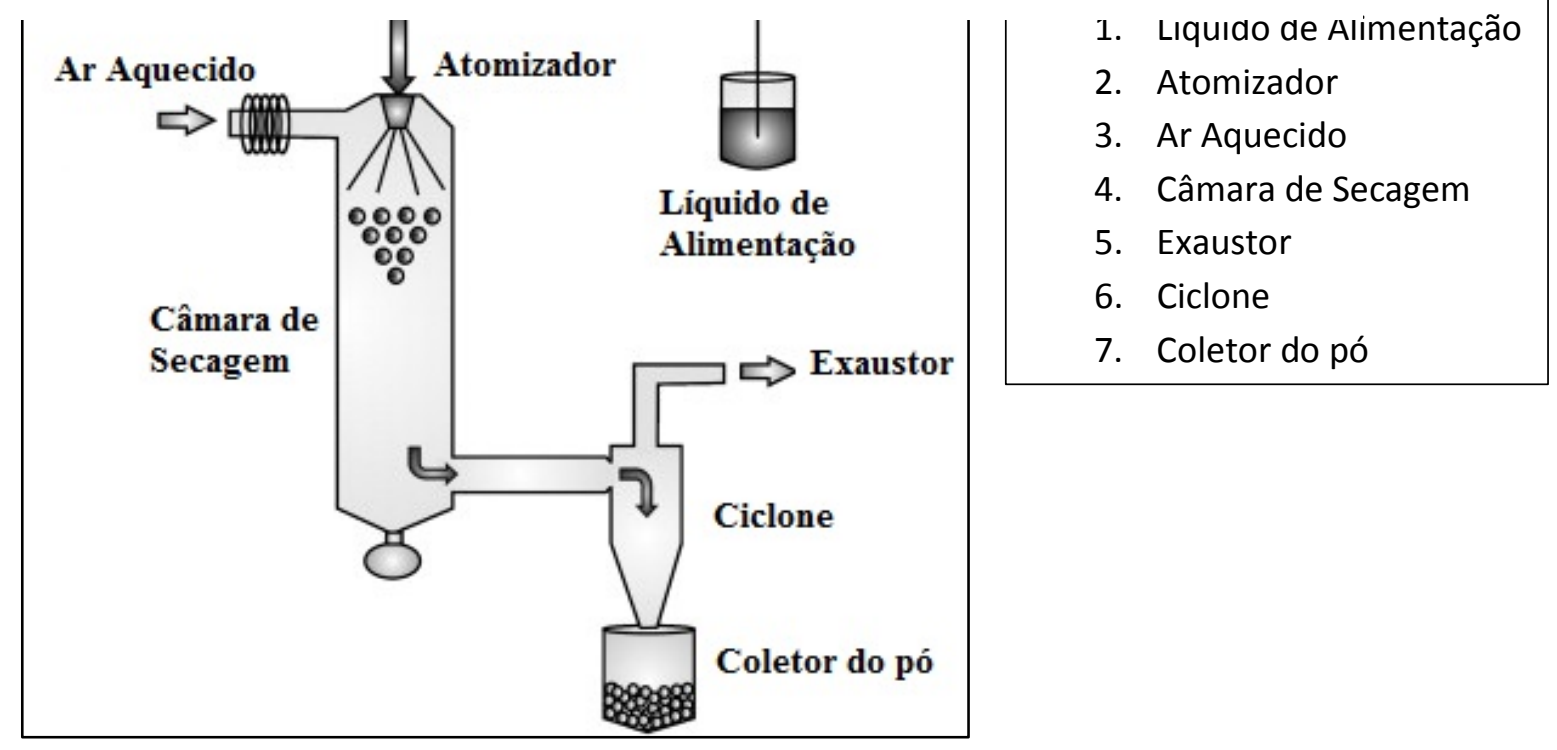

Fonte: Sosnik; Seremeta, 2015

Segundo Keshani et al., (2015), as variáveis que afetam as características do produto e que podem ser controladas são: i) propriedades do líquido de alimentação, ii) design do equipamento e iii) parâmetros do processo. 
Diversos são os parâmetros que influenciam as propriedades do pó seco produzido pelo processo spray drying (OI, 2011). A Tabela 1 fornece, resumidamente, as principais variáveis e suas influências.

\section{Tabela 1 - Influência das variáveis no Spray Dryer}

\begin{tabular}{cc}
\hline Variável & Influência \\
\hline $\begin{array}{c}\text { Vazão de alimentação } \\
\text { Densidade }\end{array}$ & $\begin{array}{c}\text { Maior concentração reduz o calor requerido pelo } \\
\text { spray dryer e forma partículas grandes } \\
\text { Vazão alta produz partículas grandes } \\
\text { Viscosidade } \\
\text { Seu efeito varia inversamente ao tamanho da } \\
\text { partícula }\end{array}$ \\
Temperatura do fluido & $\begin{array}{c}\text { O tamanho da gota varia diretamente com a } \\
\text { viscosidade da alimentação }\end{array}$ \\
Vazão do ar de secagem & $\begin{array}{c}\text { Aumento da temperatura de entrada diminui o } \\
\text { consumo de calor requerido pelo secador } \\
\text { Vazão alta, baixo tempo na câmara, } \\
\text { Temperatura do ar de secagem } \\
\text { consequentemente, alta umidade residual } \\
\text { Aumento da temperatura provoca a diminuição da } \\
\text { densidade do produto }\end{array}$ \\
\hline
\end{tabular}

Fonte: Masters, 1985

A velocidade de secagem é ligada a três fatores: a superfície de evaporação, a diferença da pressão parcial da água entre a gotícula e o ar e a velocidade de migração de água na gotícula. Pequenas gotículas formadas apresentam elevada área superficial, resultando em uma rápida evaporação de água e uma temperatura relativamente baixa, minimizando os danos térmicos ao produto (SCHUCK, 2010 apud SILVEIRA et al., 2013)

Recentemente, a engenharia de partículas tem sido usada para projetar partículas complexas para atenderem certas demandas (VEHRING; FOSS; LECHUGABALLESTEROS, 2007). Como partículas de baixa densidade, com morfologia de superfície controlada, partículas com camadas funcionais ou com dimensões menores, como nanopartículas (VEHRING; FOSS; LECHUGA-BALLESTEROS, 2007).

\subsection{Spray Dryer na indústria alimentícia}

A secagem por Spray Dryer é um processo amplamente utilizado na indústria de alimentos e, em condições ideais de processamento, tem se mostrado eficaz na obtenção de diversos produtos. É a técnica de secagem rápida, econômica e mantém as propriedades do produto (SANTOS et al., 2014; SHISHIR; CHEN, 2017).

Grande parte dos gêneros alimentícios secos comercializados é produzida através do processo de atomização por Spray Dryer. Esta técnica é a mais indicada para este tipo de 
produção pela alta sensibilidade dos produtos e pela necessidade de manter as características naturais do produto, entre elas sabor, cor, aroma, propriedades nutricionais (macro e micronutrientes) (OI, 2011). A secagem de frutas e vegetais é um processo comum e bem estabelecido na indústria alimentícia. Com a remoção da água há uma interrupção do crescimento de microrganismos e uma diminuição da deterioração do produto pelos mesmos, bem como diminuição de enzimas e reações não enzimáticas no material. Os produtos resultantes possuem excelente qualidade, isto é, baixa umidade, baixa higroscopicidade e tamanho de partícula pequeno (KARAM et al., 2016; SHISHIR; CHEN, 2017).

O seu benefício, em relação à secagem por desidratadora, está associado ao baixo tempo de exposição do produto a elevadas temperaturas, fazendo com que a temperatura no interior da partícula permaneça baixa, o que pode levar a uma menor degradação dos nutrientes do produto (AZEREDO, 2004; KESHANI et al., 2015).

Se comparado a outros métodos como a liofilização e a secagem a vácuo, a secagem por Spray Dryer é considerada mais econômica devido às baixas despesas operacionais. A técnica é de 4 a 5 vezes mais econômica do que a liofilização e 4 vezes mais econômica do que a secagem a vácuo (HAMMAMI; RENÉ, 1997; KULOZIK; FOERST, 2007 apud SHISHIR; CHEN, 2017).

Em geral, esta técnica é muito adequada tanto em laboratório quanto em escala industrial, devido à facilidade nas configurações, a capacidade de produzir pós de um tamanho específico de partículas e controle da umidade, independente da capacidade do secador. É uma operação rápida, que opera em modo contínuo, podendo ser controlado automaticamente com uma resposta rápida, ser reprodutível, ocorrer em uma única etapa e ser escalável sem a necessidade de grandes modificações (KESHANI et al., 2015; SOSNIK; SEREMETA, 2015).

Embora a secagem por Spray Dryer exija um equipamento caro e tenha um elevado desperdício de energia, a técnica permite (OI, 2011):

\footnotetext{
i) obtenção de partículas de alta qualidade, de tamanho uniforme e forma esférica,

ii) possibilidade de secar produtos à pressão atmosférica,

iii) facilidade de produzir grandes volumes em operação contínua utilizando-se equipamentos de fácil operação, e

iv) ampla aplicabilidade e flexibilidade por permitir o processamento de diversos tipos de materiais com rapidez e baixa umidade.
}

Ainda, a técnica quando voltada para produtos sensíveis ao calor pode ser melhorada com uma análise mais sistemática das formulações dos produtos em relação ao seu efeito sobre os componentes ativos, as condições de secagem e armazenamento sejam bem 
definidas. Isso pode ser realizado por procedimentos de secagem de gotículas únicas combinado com modelos preditivos que podem mapear o comportamento de secagem e a cinética de inativação das moléculas dos componentes ativos (SCHUTYSER; PERDANA; BOOM, 2012).

Estudos demonstram a eficiência do uso de Spray Dryer para a desidratação de variados tipos de alimentos. Patil, Chauhan e Singh (2014) otimizaram o processo de Spray Dryer para a produção de pó de goiaba usando as condições de temperatura de entrada de ar de $185^{\circ} \mathrm{C}$ e concentração de maltodextrina de $7 \%$, os resultados encontrados demonstram que o pó da goiaba produzido apresenta propriedades nutricionais próximas ao comercial.

Triboli et. al., (2014), testou os efeitos de aditivos na obtenção de iogurte em pó por Spray Dryer, o estudo mostrou que, dentre os aditivos testados, o Aerosil 2-4\% mostrou-se mais promissor para a melhoria do processo, apresentando melhor recuperação do produto e menor retenção do material na parede da câmara de secagem. Peng et al., (2013), avaliou os efeitos dos agentes de transporte nas propriedades físico-químicas, antioxidantes e biológicas do pó de batata doce roxa. Os resultados indicaram que os pós que foram produzidos com auxílio de um agente de transporte apresentaram melhor fluidez e propriedades de armazenamento, como baixo teor de umidade e elevada temperatura de transição vítrea, em comparação com os pós sem o uso do agente de transporte. Com relação aos antioxidantes, os pós que foram produzidos com maltodextrina e beta-ciclodextrina apresentaram as melhores taxas de retenção se comparados aos que foram utilizados apenas beta-ciclodextrina e sem agente de transporte. Além disso, os pós-produzidos com 30 g/100 g de maltodextrina e sem o uso de agente de transporte apresentaram melhores resultados na preservação fenóis, flavonoides e capacidade antioxidante, desta forma, o mesmo autor concluiu que a maldextrina é o melhor agente de transporte dentre todos os testados.

Fazaeli et. al., (2012) testou os efeitos das condições do Spray Dryer nas propriedades físicas do pó de amora. Ele observou que uma maior temperatura de entrada de ar provocou um aumento no rendimento e na solubilidade e uma diminuição na densidade, umidade e na atividade de água. Um aumento da concentração do agente de transporte proporcionou um aumento no rendimento de secagem e solubilidade, e diminuição da densidade, umidade e atividade de água. O aumento da taxa de fluxo de ar apresentou um efeito positivo no rendimento e na densidade e um efeito negativo sobre a solubilidade, umidade e atividade de água. 


\subsection{Desafios do uso de Spray Dryer na indústria alimentícia}

Um dos desafios do processo de secagem por Spray Dryer ocorre com a presença de gotículas maiores podendo fazer com que estas gotas ou aglomerados de partículas cheguem à parede da câmara antes que o processo de secagem esteja completo, resultando na aderência e formação de depósitos. A aderência de material à parede da câmara é uma das principais causas de perda de produto durante o processo de secagem (TRIBOLI et. al., 2014). O principal objetivo no controle do Spray Dryer é o de utilizar um mínimo de energia para deixar o pó com um teor de umidade abaixo da especificação e evitar que o pó adere às paredes da câmara (PETERSEN et. al., 2015)

Além de poderem ficar aderidos nas paredes da câmara de secagem durante o processo, podem ocorrer problemas de pegajosidade relacionados à baixa temperatura de transição vítrea em alguns tipos de produtos, desta forma, quando expostos às baixas temperaturas de secagem, eles estão sujeitos a sofrerem transformações, passando de um estado vítreo para um estado gomoso. Com isso, os pós-resultantes da secagem apresentam uma maior interação com a água, maior coesão e maior adesão. (ALMEIDA, 2012; VARNER, 2014).

Uma das formas para solucionar esse problema é com o uso dos chamados agentes de transportes, que consistem de carboidratos de elevado peso molecular ou proteínas específicas que tornam o produto menos higroscópico (VARNER, 2014). Os materiais transportadores mais utilizados são maltodextrina e goma árabe. No entanto, estudos recentes mostraram proteínas como isolados de proteína de soro de leite, caseinato de sódio e isolados de proteína de soja e os surfactantes de baixo peso molecular são eficientes para aumentar o rendimento do produto, mesmo em menor concentração (TONTUL; TOPUZ, 2017).

A adição de um agente de transporte também auxiliou na proteção de compostos bioativos dos alimentos, no rendimento, solubilidade, densidade aparente e reduziu a umidade e higroscopicidade (SHISHIR, CHEN, 2017).

Segundo Souza, 2014, outro desafio da técnica está relacionado com experiências em escalas industriais, devido aos seus grandes tamanhos, custos elevados e ambientes hostis de temperatura e umidade. 


\section{CONSIDERAÇÕES FINAIS}

Conforme apresentado no artigo, existem várias pesquisas demonstrando a efetividade do uso da técnica de Spray Dryer, em escala laboratorial, para a produção de pó de frutos e verduras, os quais apresentam constituintes com propriedades específicas e termo sensíveis, empregando moléculas que atuam como agentes de transporte e de encapsulação, não permitindo que as partículas permaneçam muito tempo expostas na câmara de secagem e revestem a partícula da exposição aos efeitos danosos.

A técnica de Spray Dryer é bem estabelecida em diferentes ramos da indústria de alimentos, destacando-se principalmente a de laticínios.

\section{REFERÊNCIAS}

ALEXANDRE, H. V. et. al. Cinética de secagem de abacaxi cv pérola em fatias. Revista Brasileira de Produtos Agroindustriais, Campina Grande, v.11, n.2, p.123-128, 2009. Disponível em: < http://www.deag.ufcg.edu.br/rbpa/rev112/Art1123.pdf>. Acesso em: 04 set. 2017.

ALMEIDA, F. D. L. Desidratação de suco de abacaxi probiótico por spray-dryer. 2012 Dissertação (Mestrado em Ciência e Tecnologia de Alimentos) - Universidade Federal do Ceará, Fortaleza, Ceará. Disponível em: http://www.ppgcta.ufc.br/Disserta\%E7\%E3o\%20de\%20Francisca\%20Diva\%20Lima\%20Al meida.pdf>. Acesso em: 04 set. 2017.

AZEREDO, H. M. C. de. Fundamentos de estabilidade de alimentos. Fortaleza: Embrapa Agroindústria Tropical, 195 p. 2004.

CAGLAYAN, H.; CALISKAN, H. Sustainability assessment of heat exchanger units for spray dryers. Energy. 2017. Acesso em: 04 set. 2017. DOI: 10.1016/j.energy.2017.02.097

CELESTINO, S. M. C. Princípios de secagem de alimentos. Embrapa Cerrados, Documentos 276, 51 p., 2010. Disponível em: < www.cpac.embrapa.br/download/1735/t>. Acesso em: 04 set. 2017.

CORREIA, L. F. M.; FARAONI, A. S.; PINHEIRO SANT'ANA, H. M. Effects of industrial foods processing on vitamins stability. Alim. Nutr., v.19, n.1. 2008. Disponível em: < http://serv-bib.fcfar.unesp.br/seer/index.php/alimentos/article/viewFile/204/209>. Acesso em: 04 set. 2017.

DADALI, G.; DEMIRHAN, E.; ÖZBEK, B. Effect of drying conditions on rehydration kinetics of microwave dried spinach. Food and bioproducts processing, v.86, p. 235-241, 2008. Acesso em: 04 set. 2017. DOI: 10.1016/j.fbp.2008.01.006. 
DZIEZAK, J. Yeasts and yeast derivatives: applications. Food Technology, v.41, n.2, p.122125, 1987. Disponível em: < $\quad<$ http://agris.fao.org/agrissearch/search.do?recordID=US8845404>. Acesso em: 04 set. 2017.

FAZAELI, M. et al. Effect of spray drying conditions and feed composition on the physical properties of black mulberry juice powder. Food and Bioproducts Processing, v.90, p. 667675, 2012. Acesso em: 04 set. 2017. DOI: 10.1016/j.fbp.2012.04.006.

KARAM, M. C. et al. Effects of drying and grinding in production of fruit and vegetable powders: A review. Journal of Food Engineering, 188, 32-49, 2016. Acesso em: 04 set. 2017. DOI: 10.1016/j.jfoodeng.2016.05.001

KESHANI, S. et al.. Spray drying: An overview on wall deposition, process and modeling. Journal of Food Engineering. v. 146, p. 152-162, 2015. Acesso em: 04 set. 2017. DOI: 10.1016/j.jfoodeng.2014.09.004.

MASTERS, K. Spray Drying Handbook. 4th. ed. London: George Godwin, 1985.

MASTERS, K. Spray Drying. Leonard Hill, London, 1972.

OI, R. K. Secagem da biomassa de banana verde em spray dryer. 2011. Tese (Doutorado em Engenharia Química), Faculdade de Engenharia Química, Universidade Estadual de Campinas, São Paulo.

PATIL, V.; CHAUHAN, A. K.; SINGH, R. P. Optimization of the spray-drying process for developing guava powder using response surface methodology, Powder Technology, v.253, p. 230-236, 2014. Acesso em: 04 set. 2017. DOI: 10.1016/j.powtec.2013.11.033.

PENG, Z. et al. Effect of carriers on psysicochemical properties, antioxidant activities and biological components of spray-dried purple sweet potato flours. LWT - Food Sciences \& Technology, v.51, p. 348-355, 2013. Acesso em: 04 set. 2017. DOI: 10.1016/j.lwt.2012.09.022.

PERRONE, I. T.; PEREIRA, J. P. F.; CARVALHO, A. F. Aspectos tecnológicos de fabricação de soro em pó: uma revisão, Rev. Inst. Lac. Cândido Tostes, v. 66, n. 380, p. 2330, 2011. Disponível em: < https://www.revistadoilct.com.br/rilct/article/view/165/173>. Acesso em: 04 set. 2017.

PETERSEN, L. N. et. al. Economic optimization of spray dryer operation using nonlinear model predictive control with state estimation. IFAC-PapersOnLine 48-8, 507-513, 2015. Disponível em: https://pdfs.semanticscholar.org/7470/25cdea3c19bd7d154f4872b7a319023d2949.pdf>. Acesso em: 04 set. 2017.

SANTOS, A. A. C. et al. Avaliação físico-química e comportamento higroscópico de goiaba em pó obtida por spray-dryer. Revista Ciência Agronômica, Fortaleza, v. 45, n. 3, p. 508-514, jul-set, 2014. Disponível em: < http://www.scielo.br/pdf/rca/v45n3/v45n3a10.pdf> Acesso em: 04 set. 2017. 
SCHUTYSER, M. A. I.; PERDANA, J.; BOOM, R. M. Single droplet drying for optimal spray drying of enzymes and probiotics. Trends in Food Science \& Technology, v. 27, p. 7382, 2012. Acesso em: 04 set. 2017. DOI: 10.1016/j.tifs.2012.05.006.

SHISHIR, M. R. I.; CHEN, W. Trends of spray drying: A critical review on drying of fruit and vegetable juices. Trends in Food Science \& Technology. Acesso em: 04 set. 2017. DOI: 10.1016/j.tifs.2017.05.006, 2017.

SILVA, E. S. et. al. Secagem de grãos e frutas: revisão bibliográfica, Revista Brasileira de Agrotecnologia, v.5, n.1, p. 19-23, jan./dez. 2015. Disponivel em: < http://www.gvaa.com.br/revista/index.php/REBAGRO/article/view/3683/pdf-09> Acesso em: 04 set. 2017.

SILVEIRA, A. C. P. et. al. Secagem por spray: uma revisão. Rev. Inst. Laticínios Cândido Tostes, v. 68, n. 391, p. 51-58, mar./abr., 2013. Acesso em: 04 set. 2017. DOI: 10.5935/22386416.20130020 .

SOSNIK, A.; SEREMETA, K. P. Advantages and challenges of the spray-drying technology for the production of pure drug particles and drug-loaded polymeric carriers, Advances in Colloid and Interface Science, 2015. Acesso em: 04 set. 2017. DOI:10.1016/j.cis.2015.05.003.

TONON, R. V.; BRADET, C.; HUBINGER, M. D. Aplicação da secagem por atomização para a obtenção de produtos funcionais com alto valor agregado a partir do açaí. Inc. Soc., Brasília, v. 6 n. 2, p.70-76, jan./jun. 2013. Disponível em: < http://revista.ibict.br/inclusao/article/view/1742/1948> Acesso em: 04 set. 2017.

TONTUL, I.; TOPUZ, A.; Spray-drying of fruit and vegetable juices: Effect of drying conditions on the product yield and physical properties. Trends in Food Science \& Technology, 2017. Acesso em: 04 set. 2017.DOI: 10.1016/j.tifs.2017.03.009.

TRIBOLI, E. P. D. R. et. al. Efeitos de aditivos na obtenção de iogurte em pó por meio de secagem por atomização em escala piloto, XX Congresso Brasileiro de Engenharia Química, 2014. Acesso em: 04 set. 2017. DOI: 10.5151/chemeng-cobeq2014-0271-26249-178307.

VARNER, A. S.; Modeling and optimization of the dehydration of beets for use as a valueadded food ingredient. 2014. 98 p. Dissertação (Mestrado em Ciências) - University of Maryland, Athens, Georgia. Disponível em: < https://getd.libs.uga.edu/pdfs/varner_audrey_s_201405_ms.pdf> Acesso em: 04 set. 2017.

VEHRING, R.; FOSS, W. R.; LECHUGA-BALLESTEROS, D. Particle formation in spray drying. Journal of Aerosol Science. v. 38, p. 728-746. 2007. Acesso em: 04 set. 2017. DOI: 10.1016/j.jaerosci.2007.04.005. 